\title{
Efficacy of arthroscopically placed pain catheter adjacent to the suprascapular nerve (continuous arthroscopically assisted suprascapular nerve block) following arthroscopic rotator-cuff repair
}

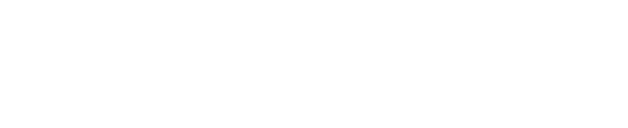

\section{Kotaro Yamakado}

Department of Orthopaedics, Fukui General Hospital, Fukui, Japan
Correspondence: Kotaro Yamakado Department of Orthopaedics, Fukui General Hospital, 58-I6-I Egami,

Fukui 910856I, Japan

Tel $+8 \mid 77659$ I 300

Fax +8I 776592538

Email yamakadok@gmail.com
Background: Rotator-cuff surgery is well recognized to be a painful procedure.

Objectives: The purpose of this study was to examine the effectiveness of an arthroscopically placed perineural catheter at the scapular notch to provide a continuous block of the suprascapular nerve (continuous arthroscopically assisted suprascapular nerve block [ca-SSNB]) following arthroscopic rotator-cuff repair (ARCR).

Materials and methods: This level II, prospective, randomized, controlled trial without postoperative blinding included 40 patients, who had a 48 -hour pain pump, with $0.2 \%$ ropivacaine infusion and a continuous rate of $3 \mathrm{~mL} /$ hour, placed via an arthroscopically placed catheter following ARCR with arthroscopic release of the superior transverse ligament: 21 patients had a ca-SSNB, and 19 patients had a continuous subacromial bursal block (SAB). The visual analog scale (at 6 hours and on the first, second, and third postoperative days) and the total number of additional pain-reduction attempts during the 3 postoperative days were calculated.

Results: The respective visual analog scale scores $(\mathrm{mm})$ obtained from the ca-SSNB and SAB groups were 62.4 and $67.6(P=0.73)$ before surgery, 9.1 and $19.4(P=0.12)$ at 6 hours after surgery, 24.4 and $44.6(P=0.019)$ on the first postoperative day, 19.4 and $40.4(P=0.0060)$ on the second postoperative day, and 18.5 and $27.8(P=0.21)$ on the third postoperative day. Total additional pain-reduction attempts recorded for the ca-SSNB and SAB groups during the 3 postoperative days were 0.3 times and 1.2 times $(P=0.0020)$, respectively.

Conclusion: ca-SSNB was highly effective in controlling postoperative pain after ARCR.

Keywords: shoulder, rotator cuff tear, postoperative pain control, continuous suprascapular nerve block, arthroscopic rotator cuff repair

\section{Introduction}

Rotator-cuff surgery is well recognized to be a painful procedure. ${ }^{1,2}$ Recent widespread use of arthroscopic techniques has decreased the level of postoperative pain; however, Wilson reported that a third of patients will experience severe pain on the first postoperative day following arthroscopic rotator-cuff repair (ARCR), even with administration of multimodal analgesia. ${ }^{2}$

Management of postoperative pain is important, and studies have shown that it allows for faster rehabilitation and recovery. An increase in pain also correlates with a delay in return to work and lower clinical scores 6 weeks after surgery. ${ }^{3}$ An increase in postoperative pain is correlated with a decrease in patient quality of recovery in the immediate postoperative period, a delayed return to work, and lower clinical scores 6 weeks after surgery. ${ }^{3,4}$ 
There are several modalities designed to decrease postoperative pain. Opioid analgesics are helpful, but there are several adverse effects associated with their use, including nausea and vomiting, pruritus, sleep disturbance, constipation, and opioid-induced hyperalgesia. ${ }^{5}$ The infiltration of local anesthetics to the subacromial bursa (SAB block) used to be a popular technique with encouraging initial results; nevertheless, recent studies have demonstrated that the technique is associated with marginally better pain relief compared with that using placebo. Consequently, this technique is not regarded as first-line therapy for pain relief associated with rotator-cuff procedures. ${ }^{1}$

Single-injection nerve blocks have an important place in the management of pain following shoulder surgery. The interscalene nerve block (ISB) is the most popular procedure, and is being widely used in combination with general anesthesia. ${ }^{1}$ The major limitation of administering a single injection (including ISB) is that the anesthetic usually has a short duration of action. Recently, several authors have recommended the continuous interscalene block (CISB) as the gold standard for most shoulder procedures. ${ }^{1,6,7}$ Numerous clinical trials have been published evaluating the efficacy of CISB for postoperative pain management. ${ }^{1}$ However, CISB techniques are more technically challenging compared with the single-shot ISB..$^{1,8-11}$ Potential side effects of ISBs, such as Horner's syndrome, hoarseness, and phrenic nerve block (diaphragmatic paralysis), might be more prolonged in the setting of CISB than in single-shot ISB, which might be more severe and distressing for patients. ${ }^{1}$

The suprascapular nerve block (SSNB) is another type of peripheral nerve block used to provide shoulder-pain relief. ${ }^{12-29}$ The suprascapular nerve (SSN) provides sensory fibers to $70 \%$ of the shoulder joint, including the superior and posterosuperior regions of the shoulder joint, the capsule, and the overlying skin. ${ }^{13}$ The single-injection SSNB is an established anesthetic technique that is safe and effective, but is limited by a short duration of action, similar to other single-injection blocks. ${ }^{1}$ Continuous SSNB theoretically prolongs the analgesia period. However, continuous SSNB is technically challenging, and very few such cases have been reported..$^{15,20,21}$

We developed a new continuous SSNB technique using a perineural catheter placed arthroscopically at the scapular notch to provide a continuous block to the suprascapular nerve. The use of arthroscopy ensures the proximity of the catheter placement to the SSN and decreases the risks related to the SSNB (ie, nerve damage by the needle, intravascular injection, and pneumothorax). The purpose of this study was to assess the analgesic efficacy of continuous arthroscopically assisted SSNB (ca-SSNB) in patients undergoing ARCR under general anesthesia. We hypothesized that ca-SSNB would reduce the level of postoperative pain.

\section{Materials and methods}

The institutional review board approved this study, and all patients participating in the study provided informed consent. From June 2010 to January 2011, patients classified according to American Society of Anesthesiologists status as class I-II were scheduled for ARCR concomitant with a transverse scapular ligament (TSL) release for SSN decompression, and were entered into this prospective randomized trial (Figure 1). Patients were randomly divided into two groups: patients who received ca-SSNB, and patients who received $\mathrm{SAB}$ with the infiltration of a continuous local anesthetic to the subacromial bursa. The following patients were excluded from the study: patients with a history of shoulder injury or shoulder surgery, those receiving daily pain medication for problems not associated with the shoulder, those with medical contraindications to regional anesthesia, and those with a subscapularis tear requiring repair. Our indications for TSL release in patients undergoing rotator-cuff repair were: 1) a large or massive rotator cuff tear, and 2) a medium tear with posterior shoulder pain and with a narrow suprascapular notch, as evidenced by three-dimensional computerized tomography.

Evaluation methods included rating via the visual analog scale (VAS; 0-100 mm) at 6 hours and on the first, second, and third postoperative days. In addition, the total number of additional pain-reduction attempts was calculated during the 3 postoperative days. The total time required for TSL release (ie, from the time when debridement medial to the acromioclavicular (AC) joint was initiated until the time

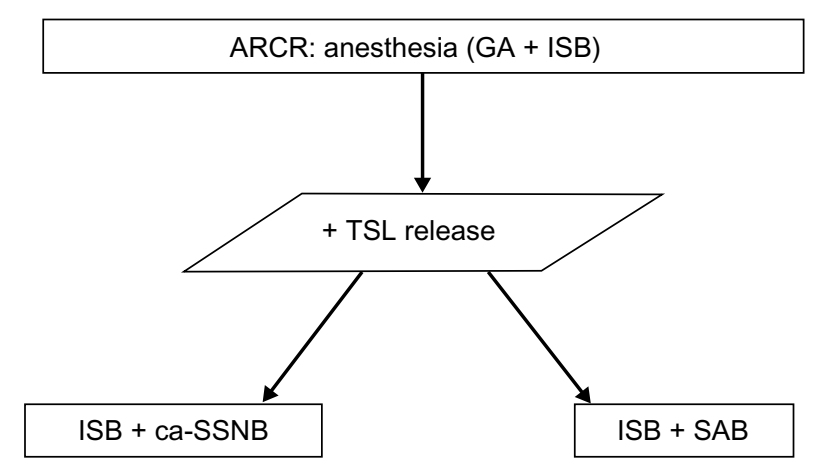

Figure I Patient flowchart.

Abbreviations: ARCR, arthroscopic rotator-cuff repair; ca-SSNB, continuous arthroscopically assisted suprascapular nerve block; GA, general anesthesia; ISB, interscalene nerve block; TSL, transverse scapular ligament; SAB, subacromial bursal block. 
of completion of the ligament release) was recorded and analyzed. Patient demographic data were compared using an unpaired $t$-test for age, weight, height, and duration of surgery, along with a $\chi^{2}$ analysis for sex ratio, the rate of arthroscopic subacromial decompression (ASAD), and the tear size. The VAS score and the elapsed time for TSL release were analyzed using the Mann-Whitney $U$ test. Statistical significance was set at $P<0.05$. Statistical analyses were performed with Microsoft Excel software (Microsoft, Redmond, WA, USA).

\section{Surgical technique}

A single surgeon performed all the surgical procedures. Patients were administered an interscalene block with $10 \mathrm{~mL}$ of $0.75 \%$ ropivacaine without the use of a nerve stimulator or ultrasound guide, but relying on palpation of the cervical muscles. No paresthesia was explored nor reported in any patient. Next, standardized general anesthesia was induced with intravenous administration of propofol (2-2.5 mg/kg). Anesthesia was maintained with oxygen and end-tidal sevoflurane, with airway management using laryngeal mask airway and spontaneous respiration. Once general anesthesia was accomplished, the patient was positioned in the beach-chair position with the arm held in flexion and with $1-3 \mathrm{~kg}$ longitudinal traction according to the patient's body weight. The superficial anatomy of the shoulder was identified, and the skin was marked to outline the clavicle, acromion, scapular spine, and the coracoid process. Two reference lines were drawn (Figure 2): one connected the medial edge of the scapular spine and the anterolateral

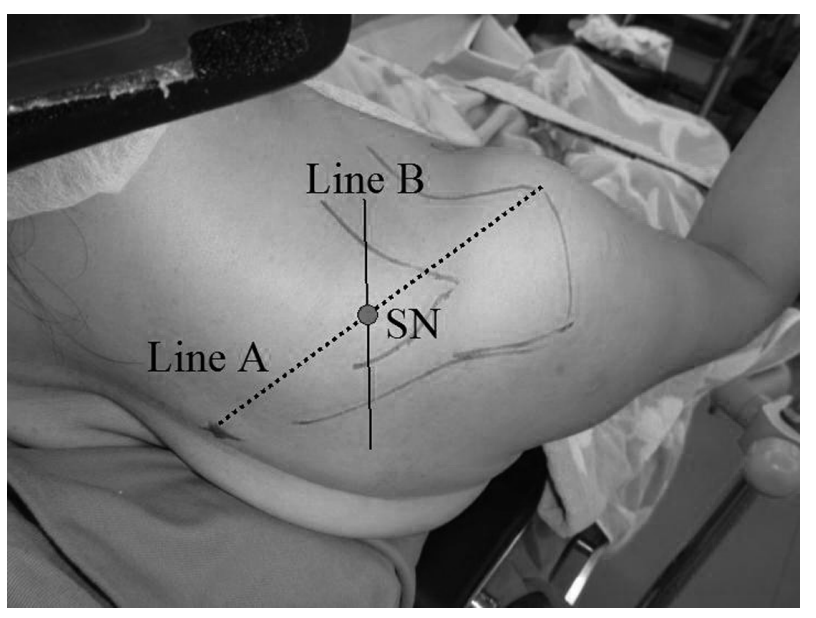

Figure 2 Drawing before the surgery. Two reference lines are drawn: one connected the medial edge of the scapular spine and the anterolateral acromion (line A), and the other was drawn to divide it midway (line B). The suprascapular nerve (SN) portal is created at the intersecting point of line A and line B. No portal should be created medially beyond this line $B$, to avoid injury to the accessory nerve. acromion (line A), and the other was drawn to divide it midway (line B). A standard viewing portal, approximately $1 \mathrm{~cm}$ medial and $2 \mathrm{~cm}$ inferior from the posterolateral corner of the acromion, was established, and the arthroscope was introduced. The arthropump was turned on, and the pressure was set at $50 \mathrm{mmHg}$. Diagnostic arthroscopy was performed, and the torn rotator cuff was repaired using suture anchors. Both single-row and double-row anchor techniques were utilized according to the tear size and torn cuff mobility. For medium tears, the double-row repair technique was utilized as long as the tendon edge was easy to approximate to the cuff footprint; otherwise, the single-row technique was utilized: one to four anchors (mean 3.0) were used for medium tears, and three to six anchors (mean 4.3) were used for large-tomassive tears.

TSL release was performed at the end of the procedure after arthroscopic rotator-cuff repair. This was performed with or without arthroscopic subacromial decompression for each patient. Viewing was performed with the $30^{\circ}$ arthroscope from the lateral portal, usually $2-4 \mathrm{~cm}$ from the lateral acromial edge in line with the bisecting line of the lateral acromial edge. The anterolateral portal was created at about $1-2 \mathrm{~cm}$ distal from the anterolateral edge of the acromion and about $2 \mathrm{~cm}$ anterior from the lateral portal if it was not yet created. By removing the soft tissue with the shaver or the radiofrequency device (VAPR ${ }^{\circledR}$; DePuy, Raynham, MA, USA) from either the posterior or anterolateral portal, the medial border of the coracoclavicular ligament was identified. Once the medial border of the coracoclavicular ligament was adequate, the $\mathrm{SN}$ portal ${ }^{25}$ was created at the intersecting point of a line linking the medial part of the scapular spine and the anterolateral edge of the acromion (line A) and another line dividing line A (line B). In addition, this line B was utilized as a medial limiting line. No portal was created beyond this line to avoid injury to the accessory nerve, which runs medial to the intersecting line between the vertebral spinous processes and the lateral tip of the acromion. ${ }^{30}$ A $5 \mathrm{~mm}$ incision was made and a smalldiameter switching rod introduced, and the TSL was palpated as a continuity of the coracoclavicular ligament. The TSL was demarcated after clarification from the surrounding fat tissue. Then, a pair of arthroscopic scissors was introduced from an accessory SSN portal, $1 \mathrm{~cm}$ lateral to the SSN portal, and the TSL was released.

Once the TSL was released, the study groups were selected by a random drawing. Each envelope contained a marker of ca-SSNB or SAB. Once a patient was randomized to a group, a 20-gauge epidural catheter was inserted either 
into the subacromial space 1-2 $\mathrm{cm}$ away from any portals through an 18-gauge spinal needle (SAB group) or into the suprascapular notch, introducing the catheter adjacent to SSN (ca-SSNB group, Figure 3, Supplementary material). After the catheter was arthroscopically confirmed to be in the accurate location, it was connected to a patient-controlled analgesia pump unit and a $200 \mathrm{~mL}$ bag of $0.2 \%$ ropivacaine. The pump was set to deliver $3 \mathrm{~mL} /$ hour, with a bolus of 10 $\mathrm{mL}$ at the end of the surgery. A nurse or a physician removed the pump at 48 hours. To relieve the extra pain, patients were instructed to use a suppository (50 $\mathrm{mg}$ diclofenac) or to request a 15-mg pentazocine injection, and the total number of attempts/requests for pain relief were recorded. Postoperative physiotherapy was identical for both the groups. The operated shoulders were protected with an UltraSling (DJO Global, Vista, CA, USA) for 4-6 weeks, depending on the tear size.

\section{Results}

A total of 42 consecutive patients were enrolled in the study. Two patients were excluded from the study because of premature pump removal. Of the 40 remaining patients, 21 received a ca-SSNB, and 19 patients had a continuous local anesthetic infiltration to the subacromial bursa (SAB group). No surgical complications were reported for any of the 40 patients. Demographic data - age, sex ratio, weight, height, duration of surgery, rate of ASAD, and tear size - were similar for both the groups (Table 1).

The following VAS scores were obtained from the caSSNB group and the SAB group, respectively: $62.4 \pm 22.3$ and 67.6 $\pm 25.9(P=0.73)$ before surgery, $9.1 \pm 15.9$ and $19.4 \pm 23.4$

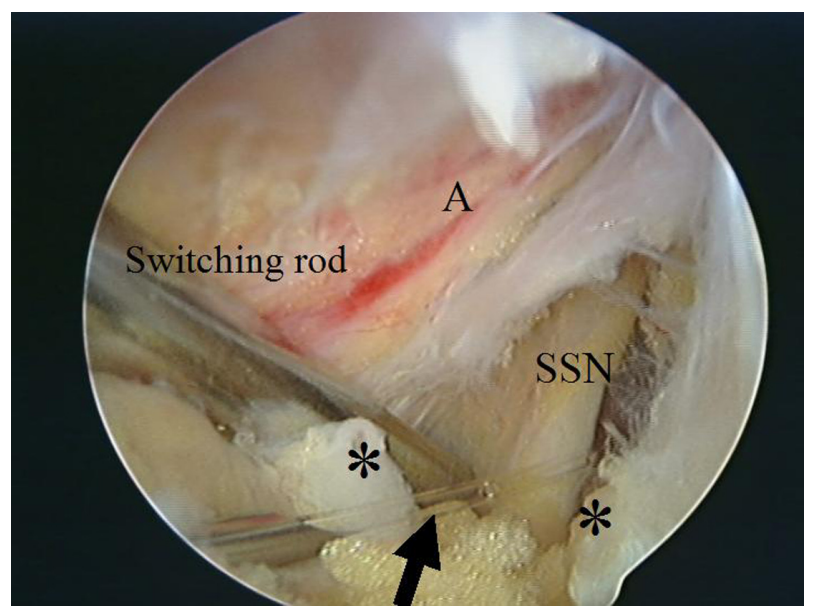

Figure $3 \mathrm{~A}$ pain catheter is set adjacently to the suprascapular nerve (SSN; continuous arthroscopically assisted suprascapular nerve block).

Notes: *Transverse scapular ligament (cut); A, suprascapular artery; the arrow indicates the epidural catheter.
Table I Patient demographics

\begin{tabular}{lll}
\hline & $\begin{array}{l}\text { ca-SSNB } \\
(\mathbf{n}=\mathbf{2} \text { I) }\end{array}$ & $\begin{array}{l}\text { SAB } \\
(\mathbf{n}=19)\end{array}$ \\
\hline Age (years) & $66 \pm 8.8$ & $59 \pm 9.1$ \\
Sex ratio (male:female) & $14: 7$ & $12: 7$ \\
Weight $(\mathrm{kg})$ & $60.4 \pm 12.0$ & $63.2 \pm 9.8$ \\
Height $(\mathrm{cm})$ & $161 \pm 0.1$ & $165 \pm 0.09$ \\
Duration of surgery (minutes) & $114 \pm 31$ & $128 \pm 22$ \\
ASAD & $18(86 \%)$ & $16(84 \%)$ \\
Tear size & & $13(68 \%)$ \\
$\quad$ Medium & $15(71 \%)$ & $6(32 \%)$ \\
\hline
\end{tabular}

Note: Data are expressed as means \pm standard deviation $\mathrm{n}(\%)$.

Abbreviations: ca-SSNB, continuous arthroscopically assisted suprascapular nerve block; $S A B$, subacromial bursal block; $A S A D$, arthroscopic subacromial decompression.

$(P=0.12)$ at 6 hours after surgery, $24.4 \pm 17.5$ and $44.6 \pm 31.9$ $(P=0.019)$ on the first postoperative day, $18.7 \pm 15.4$ and $40.4 \pm 28.6(P=0.0060)$ on the second postoperative day, and $18.5 \pm 19.8$ and $27.8 \pm 22.1(P=0.21)$ on the third postoperative day (Figure 4). The total number of additional pain-reduction attempts recorded for the ca-SSNB and SAB groups at the third postoperative day were $0.3 \pm 0.7$ times and $1.2 \pm 1.5(P=0.0020)$, respectively (Figure 5 ). The times for TSL release required in the ca-SSNB and SAB groups were 14.6 \pm 12 minutes (range 4-42 minutes) and 13.8 \pm 12 minutes (range 5-42 minutes), respectively $(P=0.76)$.

\section{Discussion}

This study revealed that ca-SSNB using an arthroscopically placed catheter decreased postoperative pain. The VAS scores at the first and second postoperative days and the total number of pain-reduction attempts were significantly lower in the ca-SSNB group than in the control group. In other words, the ca-SSNB covered the painful period from the time the single-shot ISB was no longer effective.

The innervation of the shoulder joint is primarily supplied by the suprascapular, axial, and lateral pectoral nerves. The SSN is reported to comprise $70 \%$ of the sensory innervation of the shoulder joint. The SSN originates from the upper trunk (C5, C6) of the brachial plexus, runs under the TSL at the suprascapular notch, and then supplies motor fibers to the supraspinatus and sensory fibers to the joint capsule. The nerve then runs around the inferior notch under the spinoglenoid ligament and terminates with the motor fibers to infraspinatus. There are many reports indicating that SSNB is efficient in relieving shoulder pain in many conditions, including the postoperative state. ${ }^{12-29}$ However, there are very few reports regarding the efficacy of the continuous 


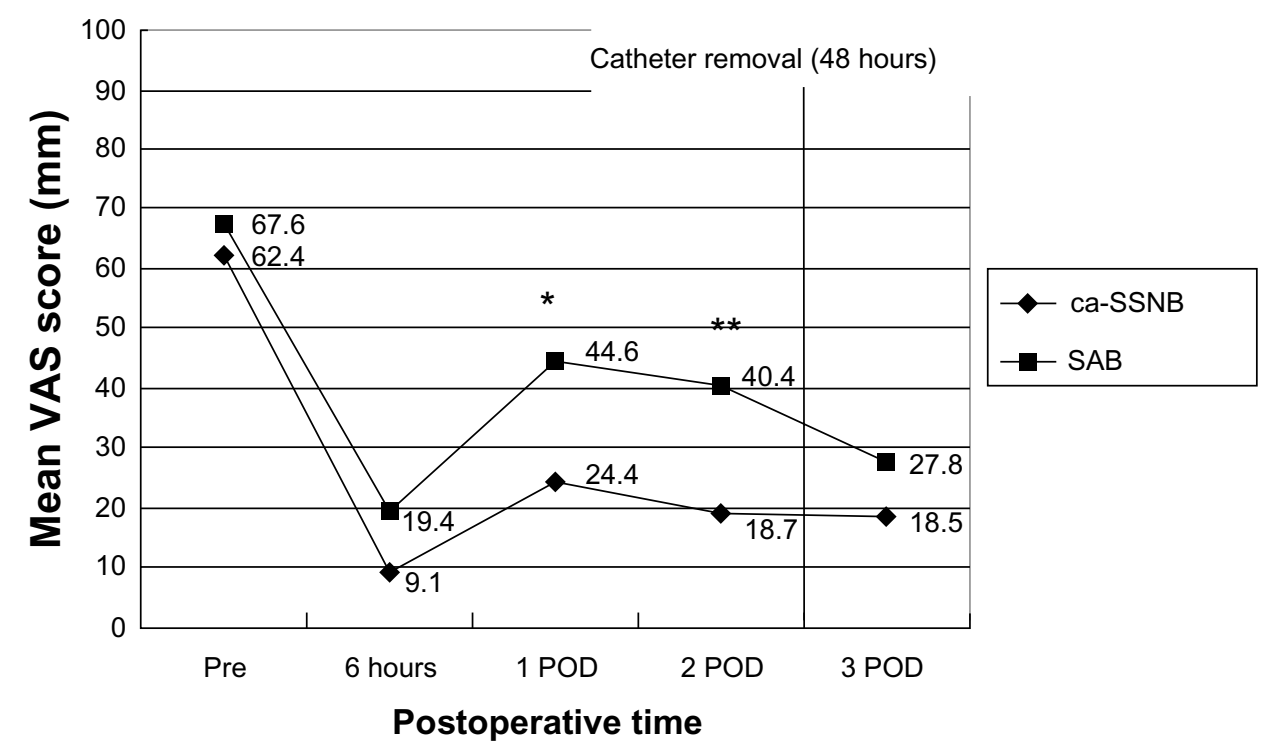

Figure 4 Visual analog scale (VAS) during the 3 postoperative days.

Notes: $* P<0.05 ; * * P<0.01$

Abbreviations: ca-SSNB, continuous arthroscopically assisted suprascapular nerve block; SAB, subacromial bursal block; POD, postoperative day; VAS, visual analog scale.

SSN block, because of technical difficulties encountered with the procedure. . $^{15,20,21}$

To our knowledge, there are no previous reports regarding a continuous SSN block via an arthroscopically placed catheter at the suprascapular notch. However, Coetzee et al described a technique utilizing an indwelling catheter placed at the spinoglenoidal notch in order to decrease postoperative pain after ARCR. ${ }^{31}$ They placed the catheter arthroscopically from the subacromial bursa at the lateral edge of the scapular spine. Contrary to our technique, they did not identify the SSN, and nor did they report their outcome, because their technique was described in a letter to the editor. ca-SNNB described here has two advantages over their technique. First, compared to the block at the spinoglenoidal notch, the

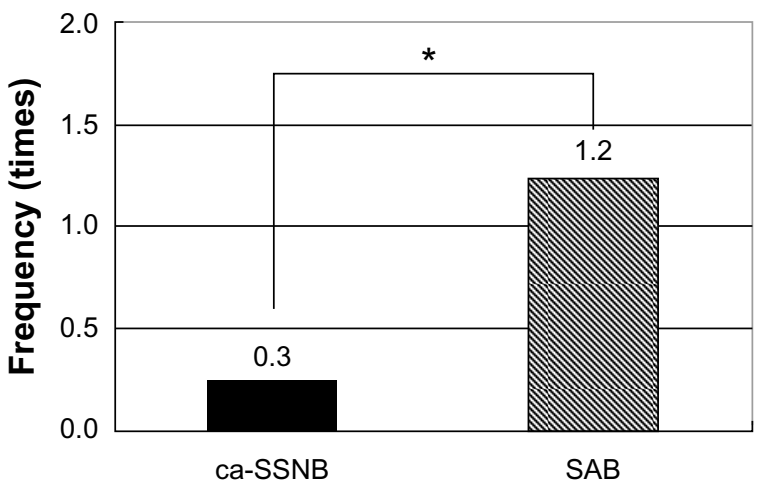

Figure 5 Total number of additional pain-reduction attempts during the 3 postoperative days.

Note: *Represents statistically significant difference $(\boldsymbol{P}=0.0020)$.

Abbreviations: ca-SSNB, continuous arthroscopically assisted suprascapular nerve block; SAB, subacromial bursal block.
SSN is blocked more proximally at the suprascapular notch. Second, our method involved placement of the catheter arthroscopically, and the SSN was identified arthroscopically as well, which ensured the proximity of the catheter to the SSN.

Nevertheless, there are several limitations to our study. First, we used this technique only in patients requiring ARCR and TSL release. The indication for SSN decompression in cases of TSL release is debatable, because SSN neuropathy associated with a rotator-cuff tear remains controversial. ${ }^{32-38}$ As SSN palsy is considered a dynamic phenomenon, ${ }^{39-41}$ existing diagnostic tools, such as electromyography and imaging studies (ie, radiography and magnetic resonance imaging), are not sufficient to demonstrate the relationship between a rotator-cuff tear and SSN palsy; therefore, the indication for performing TSL release is still under investigation. However, we believe that SSN neuropathy with a retracted rotator-cuff tear exists in significant frequency. Moreover, we believe that in cases where TSL exploration is performed, ca-SSNB should be recommended. After exploration, ca-SSNB placement is not difficult to perform, particularly with the indwelling catheter under arthroscopic visual control. Next, we evaluated each patient after surgery for only a short period; therefore, the lack of a long-term observation period precluded the long-term safety evaluation of the current technique as well as SSN decompression in terms of TSL release. Third, exploration of the TSL is a time-consuming process (ie, the mean time was 14 minutes in this study), although there is a learning curve associ- 
ated with performing such a procedure; therefore, the time required for the procedure is highly variable. We thus believe this procedure is not indicated in all ARCR cases; however, when the SSN is explored, ca-SSNB is an easy, efficient, and safe procedure under arthroscopic control. Finally, SSNB has imminent limitations in terms of the extent of blockade. As far as the extent of the block, ISB provides superior analgesia compared with SSNB. Some researchers advocate that continuous ISB is the new gold standard for shoulder surgery, considering its range and longevity. However, ISB gives motor/sensory blockade distally, which might frustrate both patients and clinicians. In addition, ISB has been inevitably linked to the development of respiratory distress because of the potential for phrenic nerve block. ${ }^{42-46}$ This is much more a concern in continuous ISB. Nevertheless, SSN does not block motor/sensory supply distally, and does not cause phrenic nerve block. We feel this is an advantage for patient control. In particular, the greatest difficulty of continuous ISB is its technical difficulty and manageability. Therefore, ca-SSNB with a single-shot ISB would be an alternative to continuous ISB.

\section{Conclusion}

ca-SSNB was highly effective in controlling postoperative pain after the anesthetic effects of ISB waned. When the SSN is explored and the TSL is released, we recommend the arthroscopic placement of a catheter adjacent to the SSN to provide effective pain relief to the shoulder.

\section{Disclosure}

The author reports no conflicts of interest in this work.

\section{References}

1. Fredrickson MJ, Krishnan S, Chen CY. Postoperative analgesia for shoulder surgery: a critical appraisal and review of current techniques. Anaesthesia. 2010;65(6):608-624.

2. Wilson AT, Nicholson E, Burton L, Wild C. Analgesia for day-case shoulder surgery. Br J Anaesth. 2004;92(3):414-415.

3. Weber SC, Jain R, Parise C. Pain scores in the management of postoperative pain in shoulder surgery. Arthroscopy. 2007;23(1):65-72.

4. Lewis RN. The use of combined suprascapular and circumflex (articular branches) nerve blocks in the management of chronic arthritis of the shoulder joint. Eur J Anaesthesiol. 1999;16(1):37-41.

5. Angst MS, Clark JD. Opioid-induced hyperalgesia: a qualitative systematic review. Anesthesiology. 2006;104(3):570-587.

6. Boezaart AP. Continuous interscalene block for ambulatory shoulder surgery. Best Pract Res Clin Anaesthesiol. 2002;16(2):295-310.

7. Nielsen KC, Greengrass RA, Pietrobon R, Klein SM, Steele SM. Continuous interscalene brachial plexus blockade provides good analgesia at home after major shoulder surgery - report of four cases. Can J Anaesth. 2003;50(1):57-61.

8. Yanovski B, Gaitini L, Volodarski D, Ben-David B. Catastrophic complication of an interscalene catheter for continuous peripheral nerve block analgesia. Anaesthesia. 2012;67(10):1166-1169.
9. Capdevila X, Jaber S, Pesonen P, Borgeat A, Eledjam JJ. Acute neck cellulitis and mediastinitis complicating a continuous interscalene block. Anesth Analg. 2008;107(4):1419-1421.

10. Sardesai AM, Chakrabarti AJ, Denny NM. Lower lobe collapse during continuous interscalene brachial plexus local anesthesia at home. Reg Anesth Pain Med. 2004;29(1):65-68.

11. Souron V, Reiland Y, Delaunay L. Pleural effusion and chest pain after continuous interscalene brachial plexus block. Reg Anesth Pain Med. 2003;28(6):535-538.

12. Lee KH, Khunadorn F. Painful shoulder in hemiplegic patients: a study of the suprascapular nerve. Arch Phys Med Rehabil. 1986;67(11):818-820.

13. Brown DE. Pain relief by suprascapular nerve block in glenohumeral arthritis. Scand J Rheumatol. 1988;17(5):411-415.

14. Emery P, Bowman S, Wedderburn L, Grahame R. Suprascapular nerve block for chronic shoulder pain in rheumatoid arthritis. $B M J$. 1989;299(6707):1079-1080.

15. Breen TW, Haigh JD. Continuous suprascapular nerve block for analgesia of scapular fracture. Can J Anaesth. 1990;37(7):786-788.

16. Meyer-Witting M, Foster JM. Suprascapular nerve block in the management of cancer pain. Anaesthesia. 1992;47(7):626.

17. Vecchio PC, Adebajo AO, Hazleman BL. Suprascapular nerve block for persistent rotator cuff lesions. J Rheumatol. 1993;20(3):453-455.

18. Ritchie ED, Tong D, Chung F, Norris AM, Miniaci A, Vairavanathan SD. Suprascapular nerve block for postoperative pain relief in arthroscopic shoulder surgery: a new modality? Anesth Analg. 1997;84(6): 1306-1312.

19. Barber FA. Suprascapular nerve block for shoulder arthroscopy. Arthroscopy. 2005;21(8):1015.

20. Borglum J, Bartholdy A, Hautopp H, Krogsgaard MR, Jensen K. Ultrasound-guided continuous suprascapular nerve block for adhesive capsulitis: one case and a short topical review. Acta Anaesthesiol Scand. 2011;55(2):242-247.

21. Mercadante S, Sapio M, Villari P. Suprascapular nerve block by catheter for breakthrough shoulder cancer pain. Reg Anesth. 1995;20(4): 343-346.

22. Dahan TH, Fortin L, Pelletier M, Petit M, Vadeboncoeur R, Suissa S. Double blind randomized clinical trial examining the efficacy of bupivacaine suprascapular nerve blocks in frozen shoulder. J Rheumatol. 2000;27(6):1464-1469.

23. Karataş GK, Meray J. Suprascapular nerve block for pain relief in adhesive capsulitis: comparison of 2 different techniques. Arch Phys Med Rehabil. 2002;83(5):593-597.

24. Harmon D, Hearty C. Ultrasound-guided suprascapular nerve block technique. Pain Physician. 2007;10(6):743-746.

25. Matsumoto D, Suenaga N, Oizumi N, Hisada Y, Minami A. A new nerve block procedure for the suprascapular nerve based on a cadaveric study. J Shoulder Elbow Surg. 2009;18(4):607-611.

26. Jeske HC, Kralinger F, Wambacher M, et al. A randomized study of the effectiveness of suprascapular nerve block in patient satisfaction and outcome after arthroscopic subacromial decompression. Arthroscopy. 2011;27(10):1323-1328.

27. Chan CW, Peng PW. Suprascapular nerve block: a narrative review. Reg Anesth Pain Med. 2011;36(4):358-373.

28. Shanahan EM, Shanahan KR, Hill CL, Ahern MJ, Smith MD. Safety and acceptability of suprascapular nerve block in rheumatology patients. Clin Rheumatol. 2012;31(1):145-149.

29. Ko SH, Kang BS, Hwang CH. Ultrasonography- or electrophysiologyguided suprascapular nerve block in arthroscopic acromioplasty: a prospective, double-blind, parallel-group, randomized controlled study of efficacy. Arthroscopy. 2013;29(5):794-801.

30. Jobe CM, Kropp WE, Wood VE. Spinal accessory nerve in a trapeziussplitting surgical approach. J Shoulder Elbow Surg. 1996;5(3): 206-208.

31. Coetzee GJ, de Beer JF, Pritchard MG, van Rooyen K. Suprascapular nerve block: an alternative method of placing a catheter for continuous nerve block. Reg Anesth Pain Med. 2004;29(1):75-76.

32. Albritton MJ, Graham RD, Richards RS 2nd, Basamania CJ. An anatomic study of the effects on the suprascapular nerve due to retraction of the supraspinatus muscle after a rotator cuff tear. J Shoulder Elbow Surg. 2003;12(5):497-500. 
33. Mallon WJ, Wilson RJ, Basamania CJ. The association of suprascapular neuropathy with massive rotator cuff tears: a preliminary report. J Shoulder Elbow Surg. 2006;15(4):395-398.

34. Costouros JG, Porramatikul M, Lie DT, Warner JJ. Reversal of suprascapular neuropathy following arthroscopic repair of massive supraspinatus and infraspinatus rotator cuff tears. Arthroscopy. 2007;23(11): 1152-1161.

35. Lafosse L, Piper K, Lanz U. Arthroscopic suprascapular nerve release: indications and technique. J Shoulder Elbow Surg. 2011; 20(Suppl 2):S9-S13.

36. Moen TC, Babatunde OM, Hsu SH, Ahmad CS, Levine WN. Suprascapular neuropathy: what does the literature show? J Shoulder Elbow Surg. 2012;21(6):835-846.

37. Shi LL, Freehill MT, Yannopoulos P, Warner JJ. Suprascapular nerve: is it important in cuff pathology? Adv Orthop. 2012;2012: 516985 .

38. Beeler S, Ek ET, Gerber C. A comparative analysis of fatty infiltration and muscle atrophy in patients with chronic rotator cuff tears and suprascapular neuropathy. J Shoulder Elbow Surg. 2013;22(11): 1537-1546.

39. Rengachary SS, Neff JP, Singer PA, Brackett CE. Suprascapular entrapment neuropathy: a clinical, anatomical, and comparative study. Part 1: clinical study. Neurosurgery. 1979;5(4):441-446.
40. Cummins CA, Messer TM, Nuber GW. Suprascapular nerve entrapment J Bone Joint Surg Am. 2000;82(3):415-424.

41. Massimini DF, Singh A, Wells JH, Li G, Warner JJ. Suprascapular nerve anatomy during shoulder motion: a cadaveric proof of concept study with implications for neurogenic shoulder pain. J Shoulder Elbow Surg. 2013;22(4):463-470.

42. al-Kaisy AA, Chan VW, Perlas A. Respiratory effects of low-dose bupivacaine interscalene block. Br J Anaesth. 1999;82(2):217-220.

43. Altintas F, Gumus F, Kaya G, et al. Interscalene brachial plexus block with bupivacaine and ropivacaine in patients with chronic renal failure: diaphragmatic excursion and pulmonary function changes. Anesth Analg. 2005;100(4):1166-1171.

44. Urmey WF, McDonald M. Hemidiaphragmatic paresis during interscalene brachial plexus block: effects on pulmonary function and chest wall mechanics. Anesth Analg. 1992;74(3):352-357.

45. Urmey WF, Talts KH, Sharrock NE. One hundred percent incidence of hemidiaphragmatic paresis associated with interscalene brachial plexus anesthesia as diagnosed by ultrasonography. Anesth Analg. 1991;72(4): 498-450.

46. Thackeray EM, Swenson JD, Gertsch MC, et al. Diaphragm function after interscalene brachial plexus block: a double-blind, randomized comparison of $0.25 \%$ and $0.125 \%$ bupivacaine. J Shoulder Elbow Surg. 2013;22(3):381-386. 


\section{Supplementary material}

Video I

Left shoulder, viewed from the lateral portal. The shaver is introduced from the suprascapular nerve (SSN) portal to demarcate the transverse scapular ligament (TSL). Thereafter, the tip of the switching rod is placed in the scapular notch to retract the SSN. A pair of arthroscopic scissors is introduced from an accessory SSN portal, $1 \mathrm{~cm}$ lateral to the SSN portal, and the TSL is released. A 20-gauge epidural catheter is inserted into the suprascapular notch, introducing the catheter adjacent to the SSN.

\section{Publish your work in this journal}

Open Access Journal of Sports Medicine is an international, peer-reviewed, open access journal publishing original research, reports, reviews and commentaries on all areas of sports medicine. The manuscript management system is completely online and includes a very quick and fair peer-review system.
Visit http://www.dovepress.com/testimonials.php to read real quotes from published authors. 